\title{
Design of an AC Servo Controller for a Dynamic Simulation Test System for Hydraulic Excavators Based on a System-on-chip Architecture
}

\author{
https://doi.org/10.3991/ijoe.v14i07.8975 \\ Yu Bo \\ Jilin Institute of Chemical Technology, Jilin City, China \\ yubo_jlict@ $126 . \mathrm{com}$ \\ Xu Chunbo \\ Jilin University, Changchun, China \\ Qiaoruibo \\ China University of Mining and Technology, Beijing, China
}

\begin{abstract}
The AC servo system of hydraulic excavator dynamic simulation test system is studied in this paper.The AC servo controller based on C8051F410 on-chip system is designed in this paper. The dynamic characteristics of AC servo are tested and the fault treatment measures of AC servo controller are improved. Comparison between traditional PID and improved PID algorithm in AC Servo Controller by dynamic Simulation Test system, the improved PID algorithm suitable for dynamic simulation test system is obtained, and the response characteristics of the improved PID algorithm to AC servo system are tested. At the same time, the reliability of AC servo controller is tested.
\end{abstract}

Keywords - motion simulation platform; AC servo; on-chip system; PID

\section{Introduction}

Compared with hydraulic servo cylinder, AC servo electric cylinder has the advantages of high transmission efficiency, no leakage, and little influence of temperature change, low noise and convenient maintenance. Based on the advantages of AC servo electric cylinder, it is applied to the dynamic platform of dynamic simulation test system of hydraulic excavator. The core components of the dynamic platform of the hydraulic excavator are AC servo controller and AC servo motor cylinder. Combining $\mathrm{AC}$ servo controller with AC servo motor cylinder,the given signal of the expansion position of the electric cylinder and the detection signal of the telescopic position of the electric cylinder can be calculated and processed.The corresponding AC servo motor operation signal is generated;make the AC servo motor moves, so that the dynamic platform system produces corresponding action. The action of AC servo motor makes the dynamic platform system produce corresponding action. 
In this paper, the dynamic simulation test system of hydraulic excavator is taken as the research object, and the AC servo controller is designed with the on-chip system as the core. The AC servo controller gives the comparison between ordinary PID, differential leading PID and incomplete differential PID. Combining with the response characteristics of AC servo, the control algorithm of differential antecedent plus incomplete differential PID is proposed.With the improved PID algorithm, the AC servo controller achieves the expected goal of the dynamic simulation test system of the hydraulic excavator.

\section{Structure of dynamic simulation test system for hydraulic excavator}

The hydraulic excavator dynamic simulation test system, as shown in Figure 1, includes the cockpit, the dynamic upper and lower platform, the 6 degree of freedom electric cylinder, the AC servo motor and the AC servo controller. The 6 degree of freedom electric cylinder combined with the upper and lower platforms can achieve 6 basic movements, that is, the straight line moving along the direction $\mathrm{XYZ}$ axis and the rotation of the three axes around the axis of the XYZ axis. Through the 6 basic movements of the platform, the complex movement of the moving platform can be realized, and the free movement of the dynamic simulation test system of the hydraulic excavator is realized

Fig. 1 is a schematic diagram of the dynamic simulation test system for a hydraulic excavator, which is a closed loop system whose core is the AC servo controller. The 6 electric cylinders (including AC servo motor) of the 6 degree of freedom motion platform are controlled by $6 \mathrm{AC}$ servo controllers. The AC servo controller can control the telescopic motion of the electric cylinder according to the position commands issued by the PC machine, and drive the dynamic simulation test system of the hydraulic excavator to produce the corresponding action. An improved PID algorithm is used in the process of control, comparing the position signals of the input 6 electric cylinders with the position signals of 6 electric cylinders, and the rapid response to the telescopic length of each electric cylinder is realized after the PID operation.

\section{Design of AC servo electronic control unit}

Based on the design of the dynamic simulation test system of hydraulic excavator, it is very important to improve the response characteristic of the system and improve the reliability of the system, design the AC servo controller reasonably. Therefore, thestability of hydraulic excavator dynamic simulation test system is closely related to the stability of AC servo controller. 


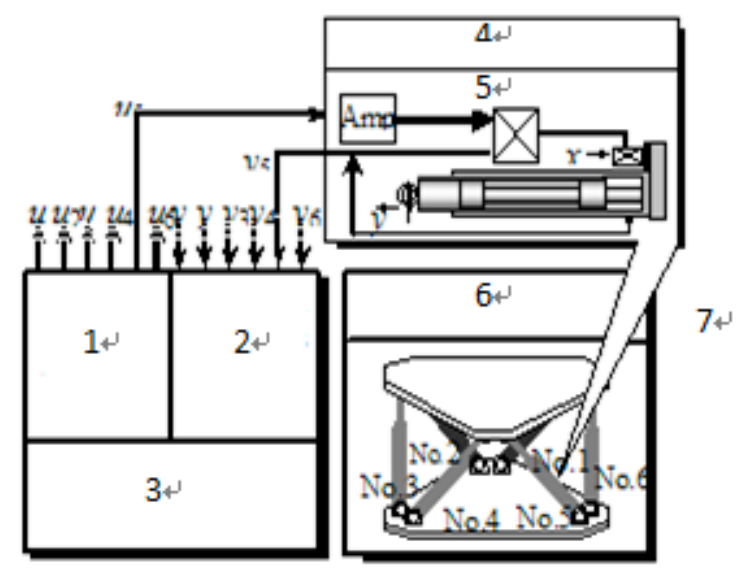

1. Servomotor speed signal 2. Photoelectric coding and proximity switch conditioning 34 . AC servo system 5.Servo motor drive 6.six degree of freedom motion platform 7.Electric cylinder

Fig. 1. Dynamic Simulation of Hydraulic Excavator

\subsection{Hardware design of AC servo controller based on chip system}

According to the interface design of AC servo controller, the AC servo controller is designed in the form of field bus ( 485 bus), and the MCU chip uses the C8051F410 model. C8051F410 is a fully integrated system type MCU on the hybrid signal. The CIP-51 kernel of the inner chip has a speed of 50MIPS, which can fully satisfy the signal acquisition of the dynamic simulation test system of hydraulic excavator and control for it.

The principle block diagram of AC servo controller based on C8051F410 on chip system, as shown in Figure 2, includes C8051F410 on chip system circuit, electric cylinder low position detection circuit, servo motor control signal circuit, RS485 interface circuit, servo motor speed signal conditioning circuit, servo motor photoelectric code disk signal circuit and power circuit. The power supply circuit adopts a stable AC/DC module, which converts the 220AC power into multiple sets of DC power supply for the system, sensors and AC servo drivers. The RS485 interface circuit is used to communicate with the PC. The low level detection circuit of the electric cylinder is used to detect the initial position of the electric cylinder. The AC servo motor control signal circuit is used to control the starting / stopping command of the servo motor. In this paper, the signal detection circuit of servo motor photoelectric encoder is simply explained.

The maximum speed of the AC servo motor used to control the dynamic simulation test system of the hydraulic excavator is 3000rpm, while the number of photoelectric code plates per cycle of the AC servo motor is 10000 yards per cycle. When the maximum speed is $3000 \mathrm{rpm}$, the time used for each pulse is calculated as (1), in which the speed is $n$ and the number of code plates is 10000 , and the minimum period of the output of a pulse in the photoelectric code disk is TImin. 


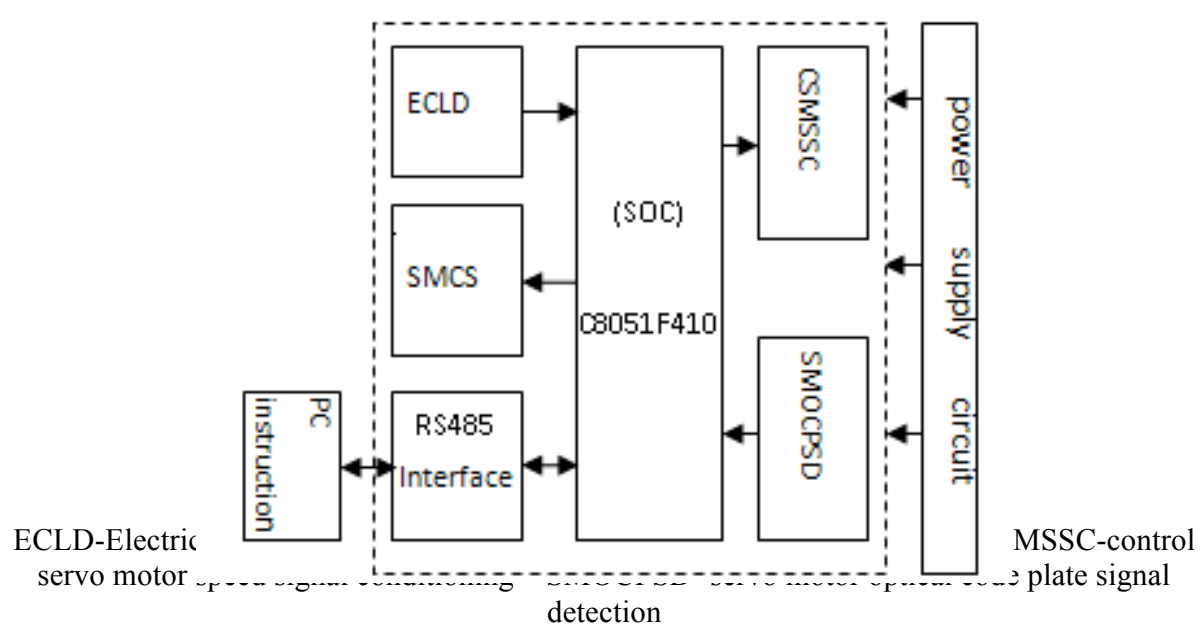

Fig. 2. AC servo control system based on c8051F410

$$
\begin{aligned}
& T 1_{\text {min }}=\frac{60}{n \times 10000}=\frac{60}{3000 \times 10000} \\
& =0.000002(\mathrm{~s})=2 * 10^{-6}(\mathrm{~s})=2(\mu \mathrm{s})
\end{aligned}
$$

When the internal clock of theC8051F410single chip is 24.5MIPS, and the C8051F410 timer / counter counts the photoelectric code disk, the maximum frequency of the C8051F410 timer / counter can reach $1 / 4$ of the system clock (24.5MIPS) frequency. The minimum period calculation of SCM sampling is shown in equation (2), where the system clock is $1 / \mathrm{T}$, and the minimum period of sampling is $\mathrm{T} 2 \mathrm{~min}$.

$$
T 2_{\min }=\frac{4}{24.5 \times 1 \times 10^{6}}=1.63 \times 10^{-7}(\mathrm{~s})=0.163(\mu \mathrm{s})
$$

Thus, T1min $>10 *$ T2min can be obtained.

Therefore, C8051F410 microcontroller can use its timer / counter to count the photoelectric encoder signal. The structure block diagram of the photoelectric code disk is shown in Figure 3.

As shown in Figure 3, the output signals of AC servo motor drivers include A, /A signals, B, /B signals, $\mathrm{Z}$ and $/ \mathrm{Z}$ signals. $\mathrm{A}$ and / $\mathrm{A}$ are the positive rotating pulse differential output signals of the $A C$ servo motor, and the $\mathrm{B}$ and $/ \mathrm{B}$ are the reverse rotating pulse differential output signals of the AC servo motor, and the $\mathrm{Z}$ and $/ \mathrm{Z}$ are the absolute zero point displacement differential signals of the AC servo motor. The photoelectric code disk A phase is 90 degrees different from the B phase. Through the 
structure shown in Figure 3, the AC servo motor is converted or reversed, and the number of positive rotating pulses and the number of reversing pulses can be counted, and the position of the electric cylinder can be known through the number of pulses.

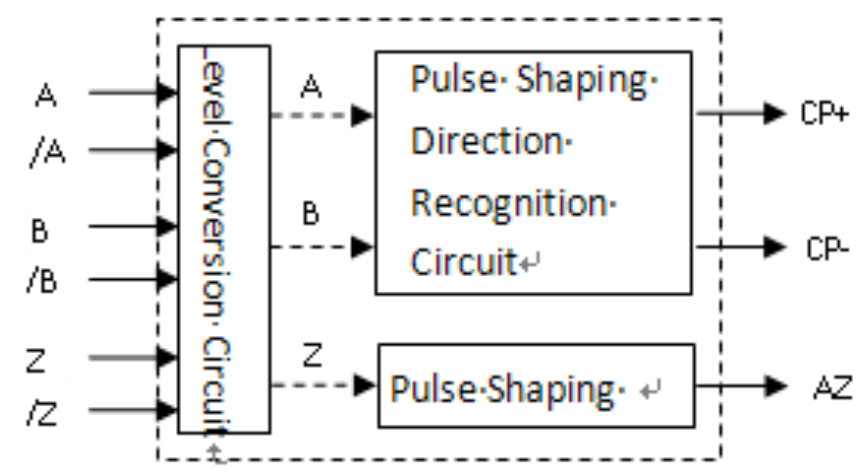

Fig. 3. Photoelectric encoder circuit

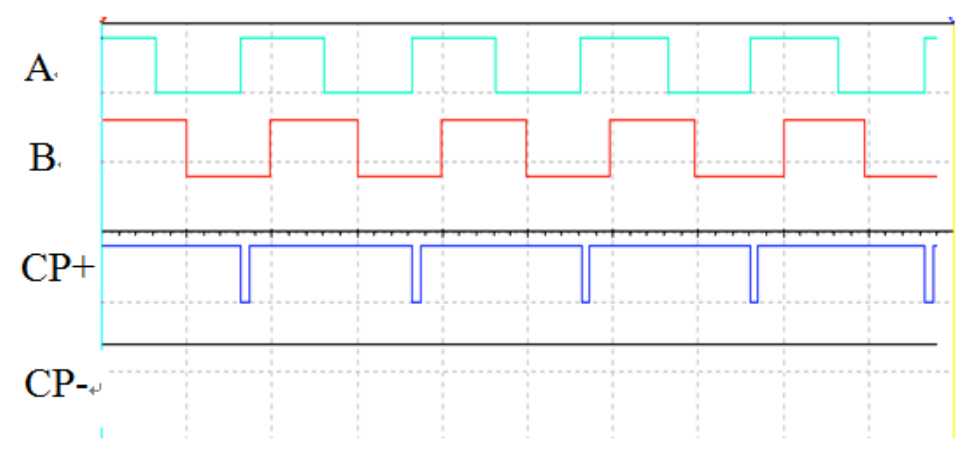

Fig. 4. Phase $\mathrm{A}$ ahead of phase $\mathrm{B} 90$ degree $\mathrm{CP}+$ and $\mathrm{CP}$ - output waveform

For the photoelectric code disk structure shown in Figure 3, when the input A phase pulse is ahead of the $\mathrm{B}$ phase pulse 90 degrees, its $\mathrm{CP}+$ and $\mathrm{CP}$ - output waveforms, as shown in Figure 5, have pulse output to the forward $\mathrm{CP}+$, and the reverse $\mathrm{CP}$ - output high level. The $\mathrm{CP}+$ and $\mathrm{CP}$ - waveforms are respectively counted on the C8051F410 microcontroller timer / counter to count the motor's positive turn, and record the number of the positive turn of the motor. Through the above judgment, the length of the electric cylinder extended is known.

Similarly, for the photoelectric code disk structure shown in Figure 3, when the input $\mathrm{B}$ phase pulse exceeds the A phase pulse at 90 degrees, its $\mathrm{CP}+$ and $\mathrm{CP}$ - output waveforms, as shown in Fig. 5, output a high level to the $\mathrm{CP}+$, and the reverse $\mathrm{CP}$ has a pulse output. The $\mathrm{CP}+$ and $\mathrm{CP}$ - waveforms are respectively counted on the C8051F410 microcontroller timer / counter to count the motor reversals, and record the number of code plates reversed by the motor. By judging above, it is known that the length of the electric cylinder is. 


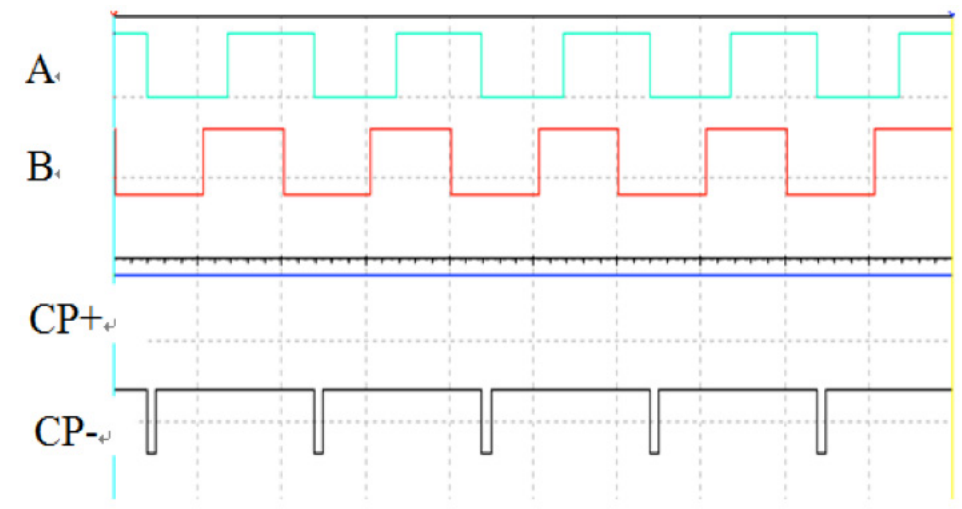

Fig. 5. Phase $\mathrm{B}$ ahead of phase A 90degree $\mathrm{CP}+$ and $\mathrm{CP}$ - output waveform

\subsection{Design of digital PID algorithm}

The traditional positional PID transfer function is shown in equation (3).

$$
U(s)=K_{P}\left[1+\frac{1}{T_{I} s}+T_{D} s\right] E(s)
$$

The traditional position PID transfer function is discretized to equation (4).

$$
u(k)=K_{p}\left\{e(k)+\frac{T}{T_{i}} \sum_{j=0}^{k} e(j)+\frac{T_{d}}{T}[e(k)-e(k-1)]\right\}
$$

The $K p$ is the ratio coefficient, the $T i$ is the integral time constant, the Td is the differential time constant, the $e(k)$ is the deviation value of the $\mathrm{k}$ sub sampling time, and the $e(k-1)$ is the deviation value at the k-1 sub sampling time, $u(k)$ is the output value of the single chip microcomputer at the time of the $\mathrm{k}$ sub sampling.

The formula (4) can see that the values of $u(k)$ are all related to the state of the past.

When the control object needs to $\operatorname{add} P, I$, and $D, e(k), \sum_{j=0}^{k} e(j) 、 \triangle e(k)$ should be added to the calculation, so the operation of MCU is much larger. The $u(k)$ output of theMCU is corresponding to the actual position of the actuator. If the $u(k)$ is misoperated,a significant change in its value will cause a large change in the position of the actuator. This situation is not allowed in actual production. When the traditional PID algorithm is used, the step response of a given electric cylinder is $150 \mathrm{~mm}$, The curve of its step response is shown in Figure 6. 


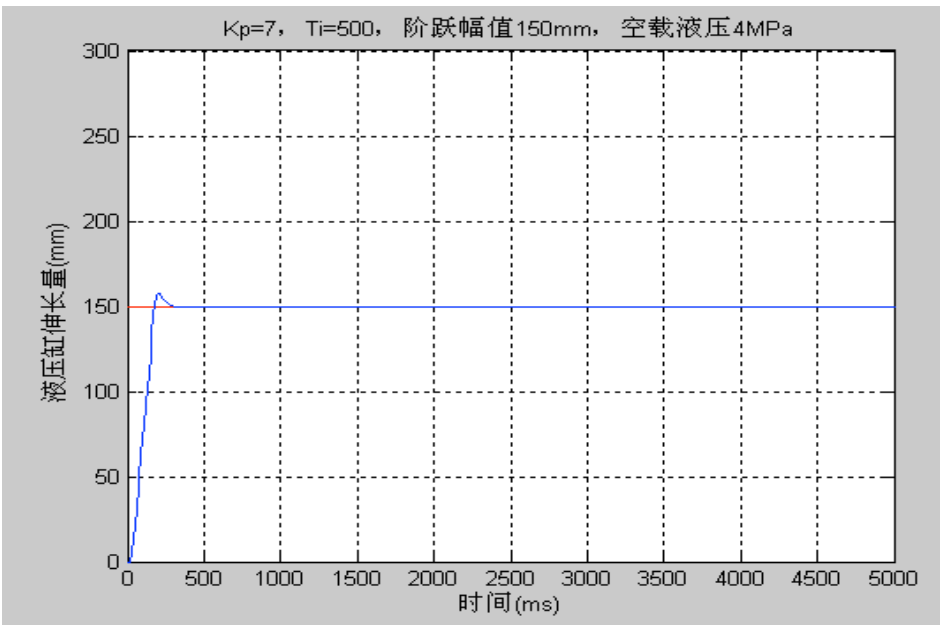

Fig. 6. Traditional PID step corresponding curve

In the traditional position PID, due to the introduction of the differentiation element, it is particularly sensitive to interference, and the output of the differential term only plays an exciting role in the first period. For a system with larger time constant, its adjustment function is small and cannot achieve the goal of precontrol error. Generally, the amplitude of $K_{p} T_{d} / T$ is generally large, and it is easy to cause data overflow. Too fast and excessive changes of differential links will also have adverse effects on executing agencies. In order to overcome these shortcomings, the differential link in the PID algorithm is transformed into a low pass filter, and the given value is filtered, as shown in equation (5).

$$
U_{D}(s)=\frac{K_{P} T_{D} s}{1+T_{F} s} R(s)
$$

The $U_{D}(s)$ in theequation (5) is discretized to equation (6).

$$
u_{d}(k)+\frac{T_{f}}{T}\left[u_{d}(k)-u_{d}(k-1)\right]=\frac{K_{p} T_{d}}{T}[r(k)-r(k-1)]
$$

Theequation (6) is finished (7.)

$$
u_{d}(k)=\frac{T_{f}}{T+T_{f}} u_{d}(k-1)+\frac{K_{p} T_{d}}{T+T_{f}}[r(k)-r(k-1)]
$$

If $T_{f} f\left(T_{f}+T\right)=\alpha$, then $T /\left(T_{f}+T\right)=1-\alpha, \quad K_{d}=K_{p} T_{d} / T, \quad u_{d}(k)$ can be reduced to form (8).

$$
u_{d}(k)=\alpha u_{d}(k-1)+K_{d}(1-\alpha)[r(k)-r(k-1)]
$$


When the $r(k)$ is given as a step signal, when $r(k)=1, k=0,1,2,3, \ldots \ldots$ Can be obtained.

$$
\begin{gathered}
u_{d}(0)=K_{d}(1-\alpha)[r(0)-r(-1)]+\alpha u_{d}(-1)=K_{d}(1-\alpha) \\
u_{d}(1)=K_{d}(1-\alpha)[r(1)-r(0)]+\alpha u_{d}(0)=\alpha u_{d}(0) \\
u_{d}(2)=\alpha u_{d}(1)=\alpha^{2} u_{d}(0) \\
u_{d}(k)=\alpha u_{d}(k-1)=\alpha^{k} u_{d}(0)
\end{gathered}
$$

By the formula (9) (12), when the incomplete differential is introduced, the pulse height of the differential output decreases in the first sampling period, and then $(\alpha<1)$.gradually attenuates according to the law of $\alpha^{k} u_{\mathrm{d}}(0)$. Therefore, incomplete differential can effectively overcome these shortcomings and has better control characteristics. The comparison between traditional PID and incomplete differential PID is shown in Figure 7.

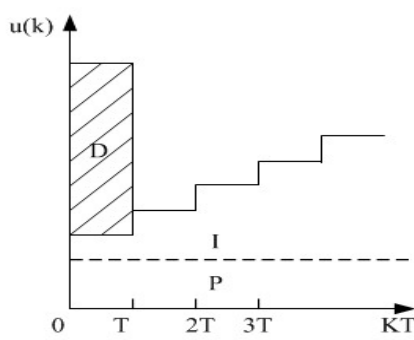

a)

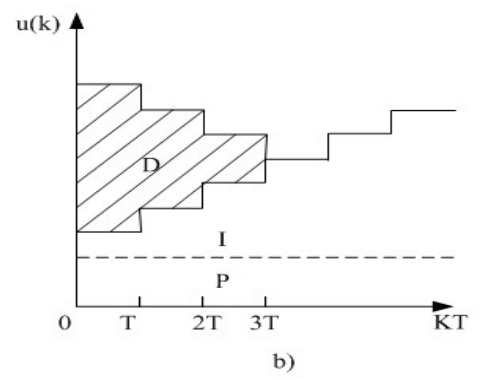

b) Incomplete differential PID

Fig. 7. Comparasion of Traditional PID with Incomplete differential PID

From Figure 7, it is known that when PID only differentiate the quantitativer(t), the change of the controlled quantity is usually relatively mild. This kind of differential control for the quantitative advance obviously improves the dynamic characteristics of the system.

According to the contrast of PID in Figure 5 and the application of AC servo in practice, the differential pre added incomplete differential position PID formula is adopted, and the improved algorithmis shown as (13).

$$
U(s)=K_{P}\left[E(s)+\frac{1}{T_{I} s} E(s)+\frac{T_{D} s}{1+T_{F} s} R(s)\right]
$$


In formula (13), the given value $\mathrm{R}(\mathrm{s})$ is used as a differential term. It is an improvement in considering that AC servo control is a servo system and the given value $\mathrm{R}(\mathrm{s})$ is a differential term, which can improve the dynamic characteristics of the system. In computer programming, the equation (13) is discretized into a differential equation (14).

$$
u(k)=K_{p}\left(e(k)+\frac{T}{T_{i}} \sum_{j=0}^{k} e(k)+u_{d}(k)\right)
$$

In formula (14), $e(k)$ is the result of the k deviation operation. $\frac{T}{T} \sum_{j=0}^{k}(e(j))$ result after $\mathrm{k}$ time deviation integral, $u_{d}(\mathrm{k})$ is the result of the $\mathrm{K}$ time adding the given value to the inertial link. $\mathrm{u}_{\mathrm{d}}(\mathrm{k})$ and $u_{d}(\mathrm{k}-1)$ can be recursively recurred by the formula (7), and $u_{d}(0)=0$.

The step response of the actual electric cylinder output location is $150 \mathrm{~mm}$, using differential first and incomplete differential PID algorithm, as shown in Figure 7.

Through the above comparison, differential step ahead and incomplete differential PID algorithm step response curve can better improve the dynamic characteristics of the system, and the overshoot is also greatly reduced.

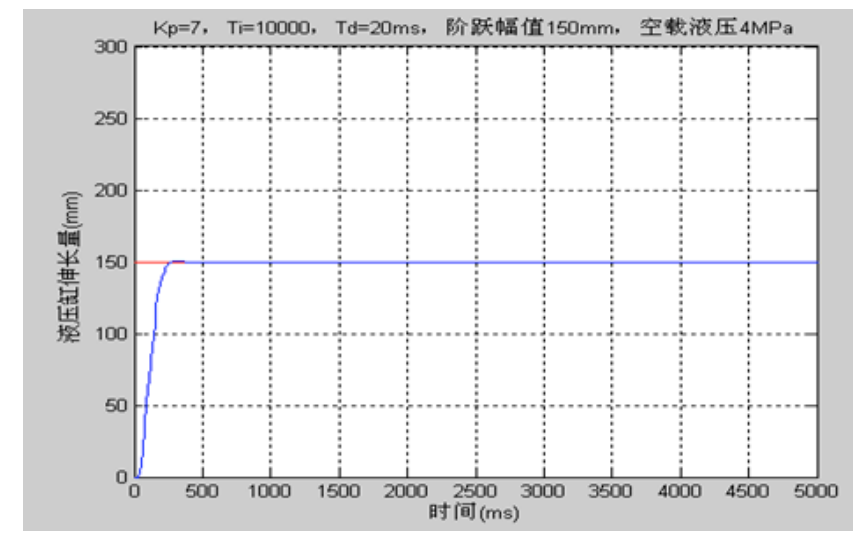

Fig. 8. Derivative ahead with incomplete different PID

\section{$4 \quad$ Analysis of experimental results}

The AC servo controller based on the C8051F410 design was introduced to the control loop of the hydraulic excavator dynamic simulation test system, which is tested according to the block diagram shown in Figure 9. The given value of the position is sent to the AC servo controller by the PC machine through the serial port and records the detected value data returned by the AC servo controller by the PC machine. 


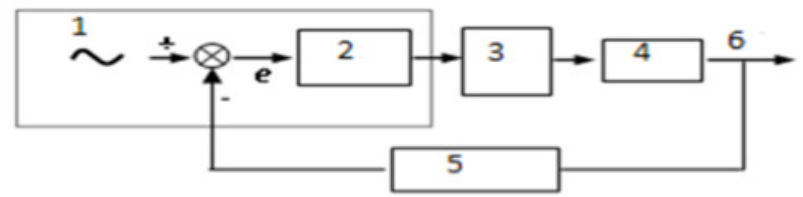

1.Input signal 2.PID algorithm 3.Servo motor 4.Electric cylinder 5.photoelectric encoder 6.Cylinder displacement

Fig. 9. Block diagram of electric servo controller

\subsection{Following characteristic test}

The input signal is given as a sine signal, and compared with the output electric cylinder displacement signal, the test waveform is shown in Figure 10, in which the dotted line is a given signal and the solid line is the following signal. The input signal is given as a random signal, which is compared with the output electric cylinder displacement signal, and the test waveform is shown in Figure 11, in which the dotted line is a given random signal and the solid line is a test signal following.

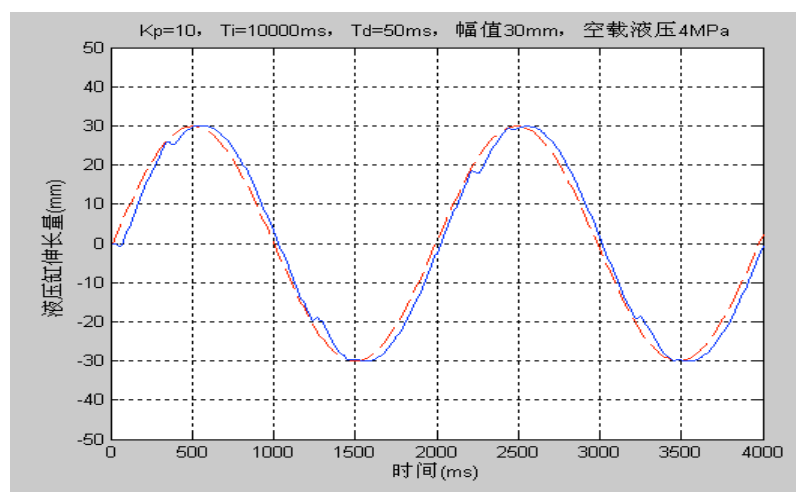

Fig. 10.Following Characteristic

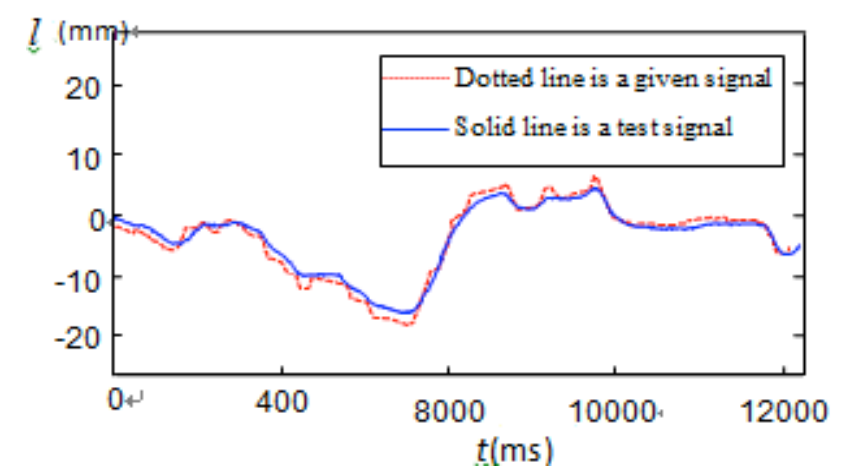

Fig. 11.Comparasion of output displacement of electric cylinder with input signal 


\subsection{Contrastive test of AC servo controller based on chip system and conventional AC servo controller}

In Figure 9, AC servo controllers are connected to the AC servo controller based on the on-chip system and the conventional AC servo controller respectively, and the step signal is added to the performance comparison test. The AC servo controller based on the on-chip system uses the differential pre - incomplete differential PID algorithm, and the conventional AC servo controller uses the traditional PID algorithm. The results of the test curves are shown in Figure 6 and figure 8 respectively. The transverse coordinates are time (unit: $\mathrm{ms}$ ), the longitudinal coordinate is the expansion length of the electric cylinder in the dynamic simulation test system of the hydraulic excavator (unit: $\mathrm{mm}$ ), and Figure 6 is the displacement curve of the electric cylinder output under the traditional PID algorithm, and the 8 is the displacement curve of the output of the electric cylinder using the differential advance and incomplete differential PID algorithm. From the result curve of the test, it can be seen that the two performance indexes of the AC servo system under the function of differential advance and incomplete differential PID algorithm are much better than those of the traditional PID algorithm.

\section{Conclusion}

1. In view of the dynamic characteristics of AC servo control system, an improved PID algorithm is proposed and applied in the project from a practical point of view.

2. The improved PID control algorithm has greatly improved the response characteristics of AC servo system.

3. The application results show that the AC servo controller based on chip system is easy to operate and has strong anti-interference capability.

\section{Reference}

[1] XIAO YINGKUI, DINGXUAN ZHAO, WANG FANGRONG: Online Simulation of Remote Motion Based on 6-DOF ParallelPlatform. Journal of Agricultural Machinery, 2005 (6): 138-140.

[2] AN ZHUOJIN, SUN DELONG, XIA XIUFENG, translation:Signal Integrated Products,Inc. [America]. Application Analysisof C8051FMicrocontroller. Beijing: Beijing University of Aeronautics and Astronautics Press,2002.

[3] AI XUEZHONG,ZHAO DING,TANG XING: Design of Electro-Hydraulic Servo Valve Controller for Engineering Robot Based on The System. Hydraulic and Pneumatic,2007(3):56-59.

[4] ZHAOJIANZHOU, ZHAO AI-LING. Design ofthe Control Circuit Based on the Position Detection of the Optical Code Disk Sensor. Electrical Automation,2006(2):75-79.

[5] HAO DINGXUAN, YANG LIFU, LI ZIYUN: Non-excavation Directional Drilling and Intelligent Control Technology at Home and Abroad. Journal of Jilin University (Engineering Edition),2005(1):44-48. 
Paper-Design of an AC Servo Controller for a Dynamic Simulation Test System for Hydraulic Exc...

[6] ZHANG HONGYAN, ZHAO DINGXUAN, ZHENG WU:Improved Fuzzy PID Control Strategy for All-Hydraulic Drillers. Journal of Wuhan University of Technology (Transportation Science Engineering),2008(8):673-676.

[7] TAOYONGHUA. NewPID ControlApplication. Machine Industry Press,Beijing,(2000).

\section{$7 \quad$ Authors}

Yui Bo (1972- ), comes from Jilin, Jilin province. Master degree, associate professor, the main research direction for computer control. (e-mail: yubo_jlict@126.com, affiliation: College of Information \& Control Engineer, Jilin Institute of Chemical Technology, Jilin City, 132022, China).

Xu Chunbo (1986- ), comes from Changchun, Jilin province. $\mathrm{PhD}$, the main research direction for robot technology and mechanical system dynamics modeling.(email:8314907@qq.com, affiliation: College of Mechanical Science and Engineering, Jilin University, Changchun, 130000, China).

Qiao Ruibo (1997- ), comes from Beijing, Beijing. Undergraduate student the main research direction forrobot technology (affiliation: School of Mechanical Electronic \& Information Engineering, China University of Mining and Technology, Beijing, Beijing, 100000, China).

Article submitted 27 March 2018. Resubmitted 25 April 2018. Final acceptance 03 June 2018. Final version published as submitted by the authors. 